\title{
MSR: Multi-Scale Shape Regression for Scene Text Detection
}

\author{
Chuhui Xue ${ }^{1}$, Shijian Lu $^{1}$ and Wei Zhang ${ }^{2}$ \\ ${ }^{1}$ School of Computer Science and Engineering, Nanyang Technological University \\ ${ }^{2}$ School of Control Science and Engineering, Shandong University \\ xuec0003@e.ntu.edu.sg, shijian.lu@ntu.edu.sg, davidzhang@sdu.edu.cn
}

\begin{abstract}
State-of-the-art scene text detection techniques predict quadrilateral boxes that are prone to localization errors while dealing with straight or curved text lines of different orientations and lengths in scenes. This paper presents a novel multi-scale shape regression network (MSR) that is capable of locating text lines of different lengths, shapes and curvatures in scenes. The proposed MSR detects scene texts by predicting dense text boundary points that inherently capture the location and shape of text lines accurately and are also more tolerant to the variation of text line length as compared with the state of the arts using proposals or segmentation. Additionally, the multi-scale network extracts and fuses features at different scales which demonstrates superb tolerance to the text scale variation. Extensive experiments over several public datasets show that the proposed MSR obtains superior detection performance for both curved and straight text lines of different lengths and orientations.
\end{abstract}

\section{Introduction}

Automated detection of various texts in scenes has attracted increasing interests in recent years due to its growing demands in many real-world applications such as image search, autonomous driving, etc. With the advance of deep neural networks (DNNs), a number of DNN based scene text detection systems have been reported and many have achieved very promising detection performance. Different scene text detection approaches have been explored which treat scene texts as one specific type of object and adapt various generic object techniques for the scene text detection task.

State-of-the-art scene text detection techniques still suffer from two typical constraints. The first is inaccurate localization due to the specific text line shapes, typically thin, having different lengths and sometimes being curved in different ways. Specifically, proposal based techniques are often at a loss in selecting anchors of appropriate aspect ratios for dealing with text lines of different lengths. Segmentation based techniques often introduce large regression errors as pixels lying around the centre of long text lines are very far from the vertices of quadrilateral regression boxes. In addition, most existing techniques generate rectangular or quadrilateral localization boxes which often include undesired image background while dealing with various curved text lines in scenes. The second is unreliable detection while dealing with texts of abnormal sizes in images. Scene texts usually have much larger scale variations as compared with generic objects [Singh and Davis, 2018], e.g. the scale ratio between the largest and the smallest texts is up to 230 times for images in the MSRA-TD500 [Yao et al., 2012]. The large scale variation often leads to miss detection for ultra-small text instances or broken/partial detection for ultra-large ones.

We design an innovative multi-scale shape regression network (MSR) that addresses both constraints at one go as illustrated in Fig. 1. Different from existing techniques, the proposed MSR regresses text pixels to the nearest text boundary points and locates scene texts by linking up the regressed text boundary points. It can thus detect scene texts of different orientations and shapes more accurately as compared with most existing techniques which produce quadrilateral vertices and often include undesired image background. In addition, MSR can locate text lines of different lengths more accurately because regressing to the nearest boundary points introduces much less regression errors as compared with regressing to the quadrilateral vertices especially for long text lines. Further, a multi-scale network is designed which empowers MSR with better tolerance to the large scale variation of texts in scenes. Specifically, it employs multiple network channels to extract and fuse features at different scales which leads to more robust detection in the presence of large text size variations. Experiments over several public datasets show that the proposed MSR is broadly applicable and achieves superior detection performance for scene texts with different orientations, shapes and lengths.

The contributions of this work are threefold. First, it proposes a novel shape regression technique to predict dense text boundary points with which scene texts of different orientations, shapes and lengths can be located accurately. Second, it proposes a multi-scale network that employs multiple network channels to extract and fuse features at different scales and demonstrates great tolerance to the large text scale variation. Third, it develops an end-to-end trainable system that achieves superior scene text detection performance over a number of public datasets with scene texts of different orientations, lengths and shapes. 


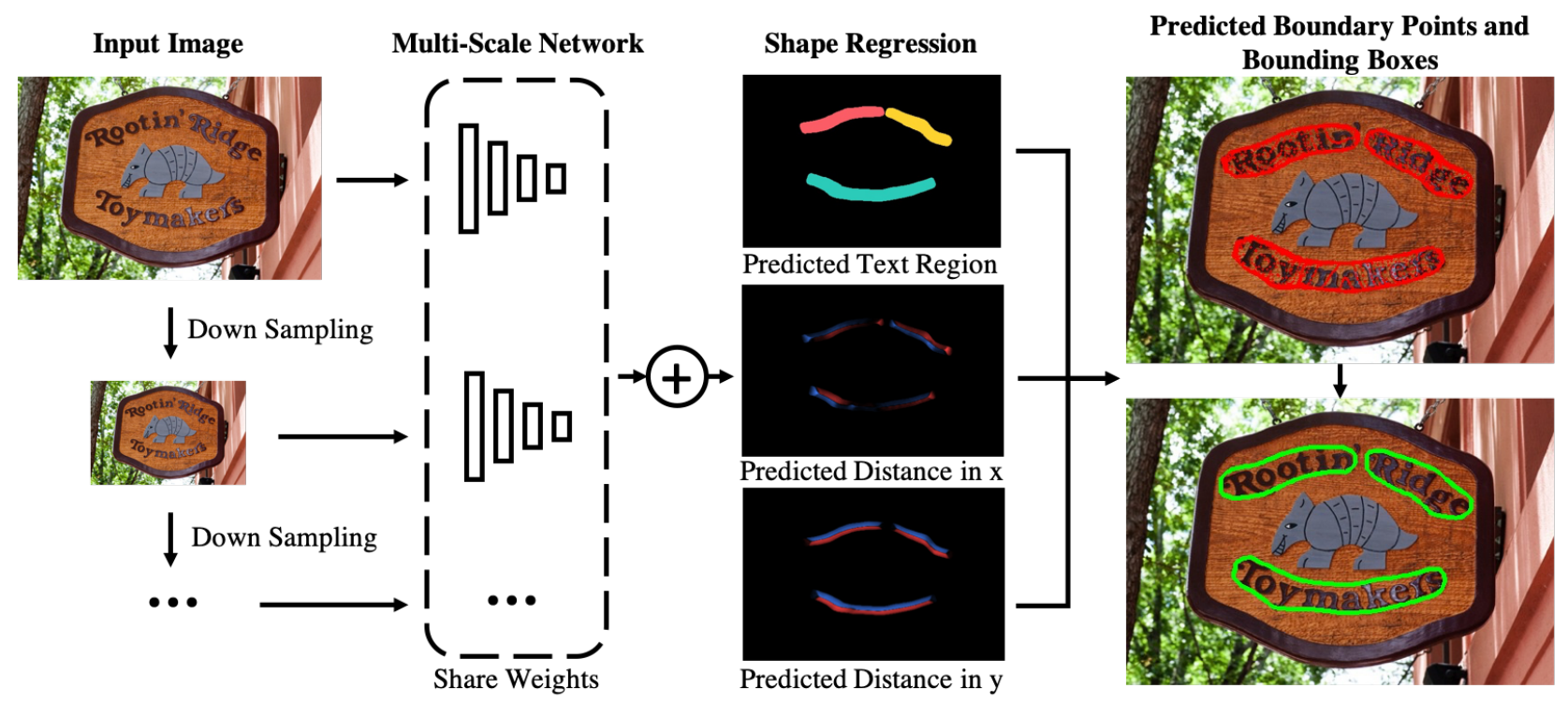

Figure 1: The framework of the proposed technique: An image and its down-scaled are fed to the multi-scale shape regression network (MSR) as input. The MSR employs multiple network channels to extract and fuse features at different scales to predict the central text regions, the distances from the central text regions to the text boundaries and dense text boundary points. Scene texts of different orientations, shapes and lengths are located by a concave polygon that encloses all boundary points of each text instance.

\section{Related Work}

\section{Scene Text Detection}

The earlier works [Tian et al., 2015; Yao et al., 2012] detect characters/words using various hand-crafted features. With the fast development of deep neural networks, a lot of CNN-based scene detection approaches have been proposed. One typical approach adapts generic object detection techniques which either leverage text-specific proposals or default boxes [Tian et al., 2016] or follow the DenseBox [Huang et al., 2015] idea by first extracting text regions and then regressing each text pixel to the vertices of localization boxes [Zhou et al., 2017; He et al., 2017; Xue et al., 2018]. In addition, some work treats scene text detection as a text segmentation problem [Deng et al., 2018], which predicts pixel-level text feature map and localizes text instances by segmenting text regions directly from the text feature map.

The recent scene text detection research predicts quadrilateral boxes of different orientations for multi-oriented text lines which still faces various problems while dealing with text lines of different shapes and lengths. Our proposed shape regression network instead predicts distances from text pixels to the nearest text boundary. It generates dense text boundary points that can be linked up to locate scene texts of very different shapes and lengths accurately.

\section{Large Scale Variation}

Two typical approaches have been explored in the era of deep learning. The first approach exploits features that are extracted at multiple network layers of different depths, instead of just using features from the last network layer which often carries large-scale global information only. For example, FPN [Lin et al., 2017] adopts a top-down network structure and treats it as a feature pyramid to make predictions at different network layers. U-Net [Ronneberger et al., 2015] extracts features from different stages of the backbone network and fuses them by up-sampling features to the same scale. The other approach employs images of different scales in network training. One typical way is to make predictions at images of different scales and then combines them as the final predictions [Hao et al., 2017]. In addition, [Singh and Davis, 2018] attempt to predict objects at specific scales where the input image is resized to the corresponding resolutions, e.g. predicting smaller objects at higher resolutions of the input image.

Our proposed multi-scale network deals with large scale variations by marrying the merits of the two state-of-the-art approaches. Specifically, it designs a new network structure that employs multiple network channels to extract and fuse features at different network stages from images of different scales simultaneously. Experiments show its superior tolerance to large object scale variations.

\section{Methodology}

We propose a novel multi-scale shape regression network for accurate detection of scene texts of different orientations, shapes and lengths as illustrated in Fig. 1. A Multi-Scale Network is designed to extract and fuse features from images of different scales. The fused features are then fed to a Shape Regression module to detect central text regions and predict distances from each central text region pixel to its nearest text boundary. This produces a set of dense text boundary points (the red points in the image in the top right corner of Fig. 1) that can be linked up to produce polygon localization boxes (the green polygon in the image in the bottom right corner). 

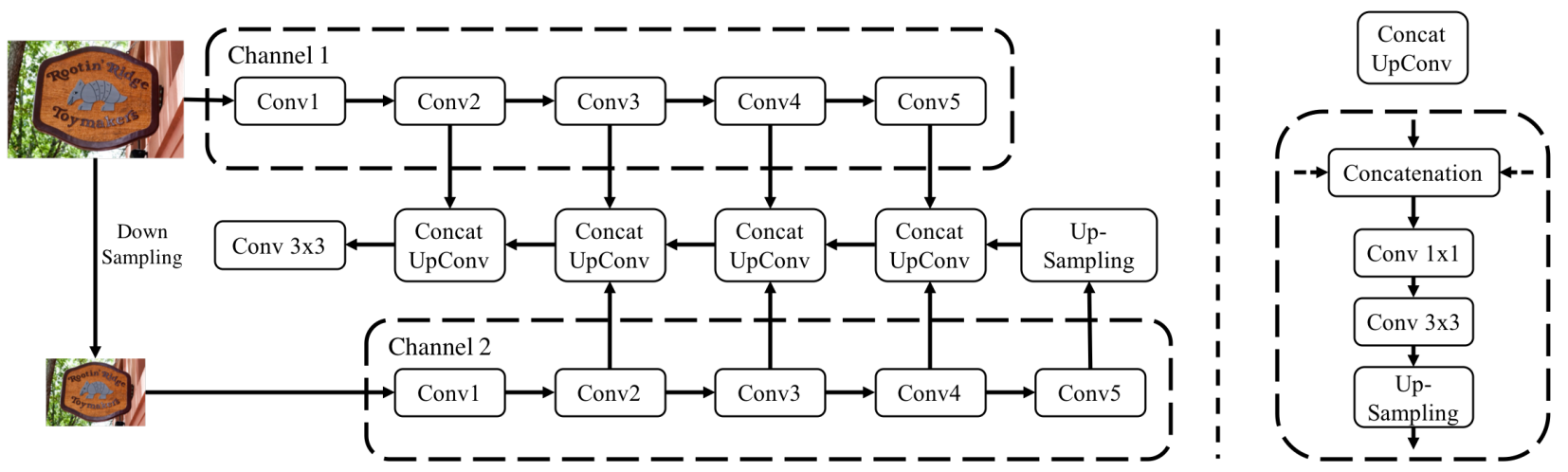

Figure 2: Structure of proposed multi-scale network (for two-scale case): Features extracted from layers Conv2 - Conv5 of two network channels are fused, where features of the same scale are fused by a Concat UpConv as illustrated and features from the deepest layer of the lower-scale channel are up-sampled to the scale of the previous layer for fusion.

\subsection{Shape Regression for Scene Text Localization}

The proposed shape regression module first performs text pixel classification and regression. The classification predicts central text regions (as illustrated in the first graph under the Shape Regression in Fig. 1) by using the fused feature map from the Multi-Scale Network. The regression predicts two distance maps (as illustrated in the second and third graphs under the Shape Regression) according to the distance between each predicted text pixel and its nearest text boundary in horizontal and vertical directions (i.e. $x$ and $y$ coordinates), respectively. Note that the central text regions are derived from the original annotation boxes in training as illustrated in Figs. $3 \mathrm{a}$ and $3 \mathrm{c}$. It is smaller than the annotation box which helps better separate neighboring words or text lines within the predicted text region map.

Each predicted text pixel will thus regress to one nearest point on text boundary which can be located by summing up the coordinates of the text pixel and the predicted distances in horizontal and vertical directions. Scene texts can thus be located by a polygon that encloses all detected text boundary points. We adopt the Alpha-Shape Algorithm [Akkiraju et al., 1995] which produces a concave polygon enclosing a set of given points. In the Alpha-Shape Algorithm, triangle edges with radius larger than alpha thresholds are removed from the delaunay triangulation graph of the text boundary points. As the radius is sensitive to size of each triangle - i.e. size of text instance, the coordinates of boundary points of each text instance are first normalized to 0 to 1 to simplify the alpha threshold selection. The polygon for each text instance is therefore first generated from the normalized boundary points and then re-sized back to the original scale.

To train the proposed shape regressor, a central text region and two distance maps are first extracted from the annotation polygon of each training text instance as illustrated in Fig. 3. Given a text annotation (may have four edges or more for curved text lines) as shown in Fig. 3a, triangulation is first performed over the annotation vertices, where each formed triangle has two vertices from the upper (or lower) side of the annotation and the third from the low (or upper) side as illustrated in Fig. 3b. For each newly formed triangle edge con-

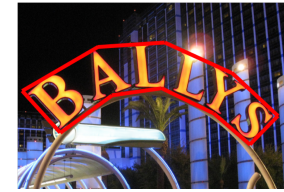

(a)

(d)

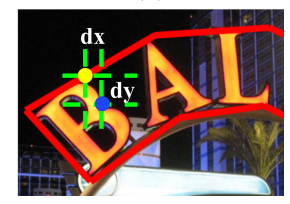

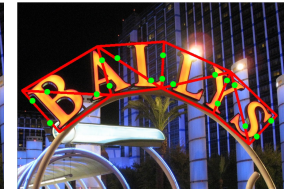

(b)

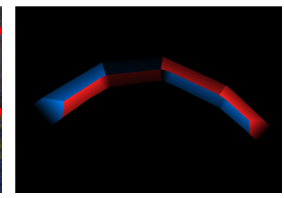

(e)

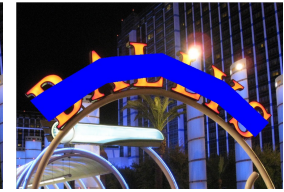

(c)

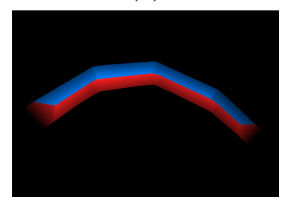

(f)
Figure 3: Illustration of ground-truth generation: Given a text annotation polygon in (a), triangulation is performed over the polygon vertices to locate the vertices (green points in (b)) of the central text region in blue color in (c). For each central-text-region pixel $t_{p}$ (in blue color in (d)), the nearest point on the text annotation box $b_{p}$ in yellow color is determined as the nearest text boundary point as shown in (d), and the distance between $t_{p}$ and $b_{p}$ is used to generate ground-truth distance maps as shown in (e) and (f)

necting the upper and lower sides of the text annotation, two points at $25 \%$ distance from each end are determined which form the vertices of the central text region as illustrated in Figs. $3 \mathrm{~b}$ and $3 \mathrm{c}$. For each pixel $t_{p}$ in the central text region, the nearest point $b_{p}$ on the text annotation lines can be located (the yellow-color point in Fig. 3d), and the distances between $t_{p}$ and $b_{p}$ can then be determined to generate the distances maps in $x$ and $y$ directions as illustrated in Fig. 3e and 3f.

\subsection{Multi-Scale Multi-Stage Detection Network}

The proposed network adopts a multi-channel structure to accommodate images of different scales. Given a training image, it is first re-sampled by a factor of 2 and produces multiple re-scaled images. The training image and the re-scaled are then fed to multiple network channels for feature extraction. Fig. 2 shows one network structure that uses two channels for the original training image and the half-scaled (this network is adopted in our implemented scene text detection system). 
Proceedings of the Twenty-Eighth International Joint Conference on Artificial Intelligence (IJCAI-19)

\begin{tabular}{|c|c|c|c|c|c|c|}
\hline & \multicolumn{3}{|c|}{ CTW1500 } & \multicolumn{3}{c|}{ Total-Text } \\
\hline Methods & Precision & Recall & F-score & Precision & Recall & F-score \\
\hline \hline SegLink [Shi et al., 2017] & 42.3 & 40.0 & 40.8 & 30.3 & 23.8 & 26.7 \\
\hline CTD+TLOC* [Yuliang et al., 2017] & 77.4 & 69.8 & 73.4 & - & - & - \\
\hline Mask TextSpotter*[Lyu et al., 2018a] & - & - & - & 69.0 & 55.0 & 61.3 \\
\hline TextSnak**[Long et al., 2018] & 67.9 & $\mathbf{8 5 . 3}$ & 75.6 & 82.7 & 74.5 & 78.4 \\
\hline \hline EAST(Baseline) [Zhou et al., 2017] & 78.7 & 49.1 & 60.4 & 50.0 & 36.2 & 42.0 \\
\hline Ours* & $\mathbf{8 5 . 0}$ & 78.3 & $\mathbf{8 1 . 5}$ & $\mathbf{8 3 . 8}$ & $\mathbf{7 4 . 8}$ & $\mathbf{7 9 . 0}$ \\
\hline
\end{tabular}

Table 1: Experimental results over the curved-text-line datasets CTW1500 and Total-Text, where methods with '*' specifically address curved text lines. Results of SegLink and EAST are taken from [Long et al., 2018].

Within each network channel, image features are extracted at multiple network stages to capture details at different levels. At the end, object features extracted from multiple network channels and multiple network stages (within each channel) are fused and fed to the Shape Regression module which will predict the central text regions and distance maps.

The simultaneous feature learning from images of different scales at different network stages is a challenging task as features from the same network stage of different network channels have different scales. Fig. 2 illustrates how our proposed network architecture addresses this challenge. As Fig. 2 shows, features from Conv 5 of Channel 2 are firstly upsampled by a factor of 2 so that they will have the same scale as features from Conv 5 of Channel 1 (and so Con 4 of Channel 2). A Concat UpConv module is exploited for feature fusion, which first concatenates features from three network stages and then up-samples the concatenated features by a factor of 2 (after a $1 \times 1$ convolution and a $3 \times 3$ convolution) [Zhou et al., 2017] and finally passes the up-sampled feature to the earlier network stage for further feature fusion. Note we only use features from stages from Conv2 to Conv 5 as those from Convl are too low-level.

The proposed network improves the detection of scene texts of very different sizes from two aspects. On the one hand, the multi-stage design improves the prediction of details at different levels by fusing local and global features effectively. On the other hand, the multi-scale design extracts features from text images of different scales which addresses the large text size variation directly.

\subsection{Network Training}

The proposed multi-scale shape regression network takes the original training image and the corresponding central text regions and distances maps as inputs. The training aims to minimize the following multi-task loss function:

$$
\mathcal{L}=\mathcal{L}_{c l s}+\lambda * \mathcal{L}_{\text {reg }}
$$

where $\mathcal{L}_{c l s}$ and $\mathcal{L}_{\text {reg }}$ refer to the loss of classification (for prediction of central text regions) and regression (for prediction of distances to the nearest text boundary), respectively. $\mathrm{Pa}-$ rameters $\lambda$ is the weight to balance the two losses which is empirically set at 1.0 in our implemented system.

The prediction of central text regions is actually a pixelwise binary classification problem. We adopt the Dice Coef- ficient loss that is defined by:

$$
\mathcal{L}_{c l s}=\frac{2 *|G \cap P|}{|G|+|P|}
$$

where $G$ and $P$ refer to the ground-truth central text region and the predicted central text region, respectively.

The prediction of distances from central text pixels to the nearest text boundary is a regression problem. We define the regression loss based on the Smooth L1 loss [Girshick, 2015]:

$$
\begin{aligned}
\mathcal{L}_{\text {reg }} & =0.5 * \sum_{k} \text { Smooth }_{L 1}\left(x_{k}-x_{k}^{*}\right) \\
& +0.5 * \sum_{k} \text { Smooth }_{L 1}\left(y_{k}-y_{k}^{*}\right)
\end{aligned}
$$

where $x$ and $y$ denote ground-truth distances in the horizontal and vertical directions, respectively, and $x^{*}$ and $y^{*}$ denote the correspondingly predicted distances. The $S$ moot $h_{L 1}$ denotes the standard Smooth L1 loss.

\section{Experiments}

\subsection{Datasets}

SynthText [Gupta et al., 2016] contains more than 800,000 synthetic scene text images most of which are at word level with multi-oriented rectangular annotations.

CTW1500 [Yuliang et al., 2017] consists of 1,000 training images and 500 test images that contain 10,751 multioriented text instances of which 3,530 are arbitrarily curved. Each text instance is annotated at text-line level by using 14 vertices, where texts are largely in English and Chinese.

Total-Text [Ch'ng and Chan, 2017] has 1,255 training images and 300 test images where texts are all in English. It contains a large number of multi-oriented curved text instances each of which is annotated at word level with a polygon.

MSRA-TD500 [Yao et al., 2012] consists of 300 training images and 200 test images. All captured text instances are printed in English and Chinese which are annotated at textline level by using best-alighed rectangles.

ICDAR2015 [Karatzas et al., 2015] has 1000 training images and 500 test images which are collected by Google Glass and suffers from low resolution and motion blur. All text instances are annotated at word level using quadrilateral boxes. 
Proceedings of the Twenty-Eighth International Joint Conference on Artificial Intelligence (IJCAI-19)

\begin{tabular}{|c|c|c|c|c|c|c|c|}
\hline & \multicolumn{3}{|c|}{ ICDAR2015 } & \multicolumn{3}{c|}{ MSRA-TD500 } \\
\hline Methods & Prediction & Recall & F-score & FPS & Prediction & Recall & F-score \\
\hline \hline SegLink [Shi et al., 2017] & 73.1 & 76.8 & 75.0 & - & 86.6 & 70.0 & 77.0 \\
\hline PixelLink [Deng et al., 2018] & 85.5 & $\mathbf{8 2 . 0}$ & $\mathbf{8 3 . 7}$ & 3.0 & 83.0 & 73.2 & 77.8 \\
\hline TextSnake* [Long et al., 2018] & 84.9 & 80.4 & 82.6 & 1.1 & 83.2 & 73.9 & 78.3 \\
\hline Mask TextSpotter* [Lyu et al., 2018a] & 85.8 & 81.2 & 83.4 & 4.8 & - & - & - \\
\hline RRD [Liao et al., 2018] & 85.6 & 79.0 & 82.2 & $\mathbf{6 . 5}$ & 87.0 & 73.0 & 79.0 \\
\hline Lyu et al. [Lyu et al., 2018b] & $\mathbf{9 4 . 1}$ & 70.7 & 80.7 & 3.6 & $\mathbf{8 7 . 6}$ & 76.2 & 81.5 \\
\hline \hline EAST(Baseline) [Zhou et al., 2017] & 80.5 & 72.8 & 76.4 & $\mathbf{6 . 5}$ & 87.3 & 67.4 & 76.1 \\
\hline Ours* & 86.6 & 78.4 & 82.3 & 4.3 & 87.4 & $\mathbf{7 6 . 7}$ & $\mathbf{8 1 . 7}$ \\
\hline
\end{tabular}

Table 2: Experimental results over the straight-text-line datasets ICDAR2015 and MSRA-TD500, where methods with '*' specifically address curved text lines. Only single-scale testing results are listed for ICDAR2015 dataset for fair comparison.

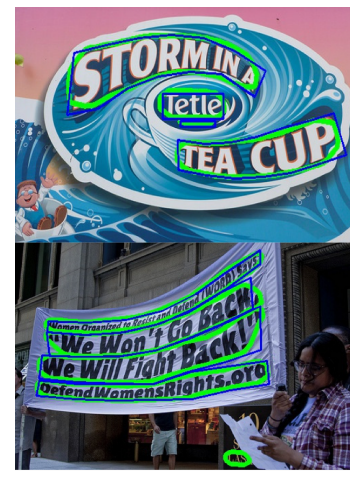

(a)

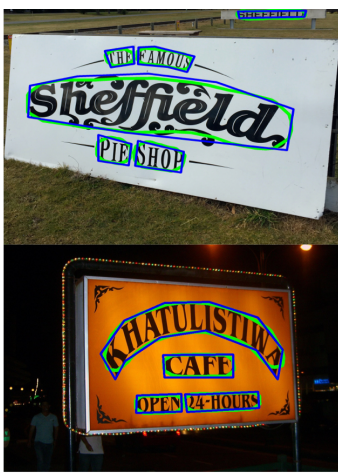

(b)

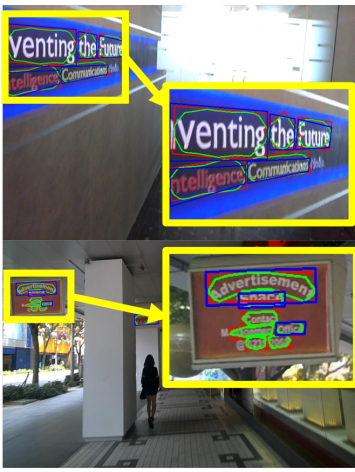

(c)

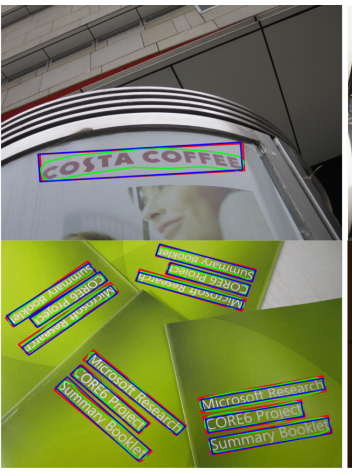

(d)

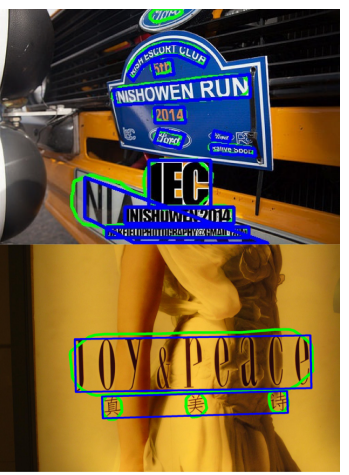

(e)

Figure 4: Illustration of the proposed scene text detection method: Sample images in (a)-(d) are selected from CTW1500, Total-Text, ICDAR2015 and MSRA-TD500 respectively, where green boxes show polygon localization boxes by our proposed method and blue boxes show ground-truth boxes. For images with straight text lines in (c) and (d), red quadrilateral boxes are derived from the polygon localization boxes for the evaluation purpose. A few typical unsuccessful cases are given in (e).

\subsection{Implementation Details}

The proposed technique is implemented using Tensorflow on a regular GPU workstation with 2 Nvidia Geforce GTX 1080 Ti. The network is optimized by Adam optimizer [Kingma and $\mathrm{Ba}, 2014$ ] with a starting learning rate of $10^{-4}$. The network is pre-trained on the SynthText, which is then fine-tuned by using the training images of each evaluated dataset with a batch size of 10. ResNet-50 [He et al., 2016] is used as the network backbone.

\subsection{Experimental Results}

The proposed technique has been evaluated quantitatively and qualitatively over four public datasets as shown in Tables 1 and 2 and Fig. 4. It has also been analyzed through ablation studies as shown in Table 3 and Fig. 5.

\section{Texts in Different Orientations and Shapes}

The proposed technique has been evaluated over the datasets CTW1500 and Total-Text where many scene texts were captured in different orientations, shapes and lengths. The purpose is to study how the proposed technique performs under the presence of many different text appearances.

As Table 1 shows, the proposed method achieves f-scores of $81.5 \%$ and $79.0 \%$ on datasets CTW1500 and Total-Text in single-scale testing mode which are significantly higher than state-of-the-art methods that didn't specifically address curved text lines. In particular, it outperforms the best fscore by 5.9 for CTW1500 with annotations at text-line level, demonstrating its superiority in dealing with text lines of different lengths. In fact, the proposed method is capable of dealing with text lines of very different lengths as illustrated in Fig. 4 because it regresses to the nearest text boundary instead of the four quadrilateral vertices. For Total-Text with scene texts annotated at word level, our proposed method also achieved state-of-the-art performance, demonstrating its superiority in dealing with curved texts with limited length variations. In addition, the proposed method is on par with or significantly outperform the very recent methods that specifically addressed curved text lines.

\section{Texts in Different Orientations and Lengths}

The proposed method is also evaluated over ICDAR2015 and MSRA-TD500 where most scene texts are straight but in different orientations with annotations at word and text-line level. We derive an oriented rectangular box from each determined concave polygon as illustrated in the second row of Fig. 4 (highlighted in red-color boxes) for overlap computation in evaluations. Table 2 shows experimental results and comparisons with state-of-the-art techniques.

For MSRA-TD500, the proposed method achieves state- 


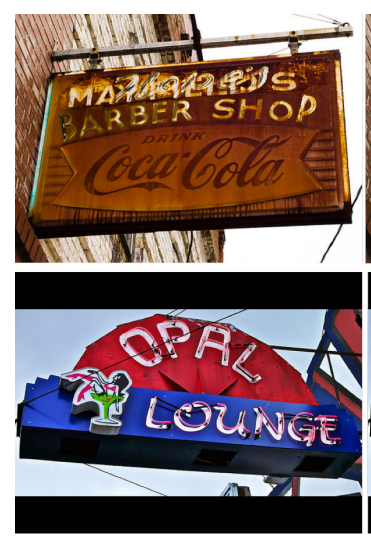

(a)

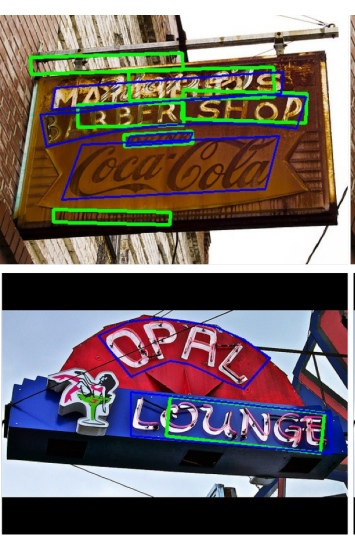

(b)

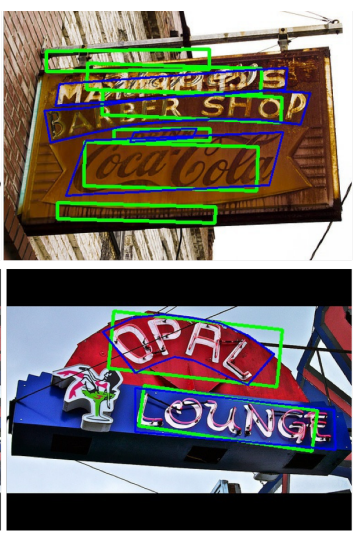

(c)

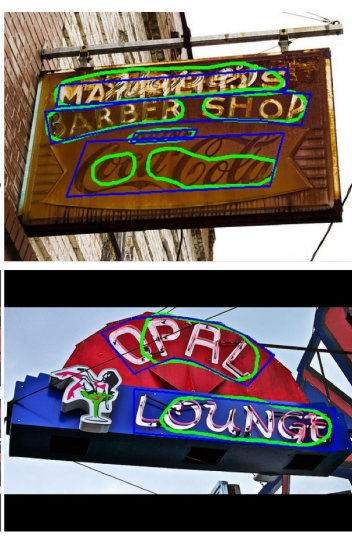

(d)

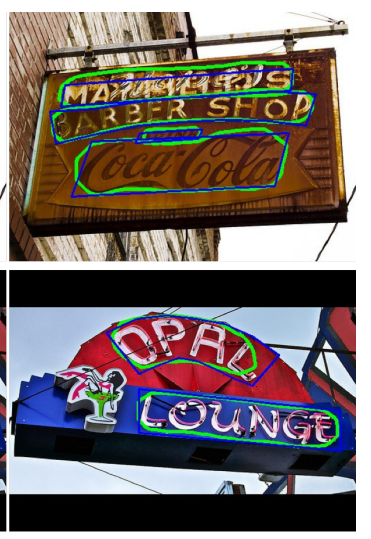

(e)

Figure 5: Ablation study of the proposed technique: For sample images from CTW1500 in (a), the green-color boxes in (b-e) show scene text detection by using the 'Baseline', 'Baseline+Multi-Scale', 'Baseline+Shape Regression' and 'Multi-Scale Shape Regression', respectively. The ground truth are shown in blue-color boxes.

of-the-art f-score, demonstrating it superior capability in dealing with straight text lines of different orientations and lengths, the subject that has been studied for many years in the scene text detection community. In fact, it outperforms most state-of-the-art methods that predict rectangular boxes which are more suitable for straight text lines. Further, it outperforms TextSnake, a very recent method that specifically addressed curved text lines, by up to 3.4 in f-score. For ICDAR2015, our approach achieves competitive performance in both accuracy and speed at single-scale testing mode.

\subsection{Ablation Study}

The proposed multi-scale shape regression network consists of two innovative components, namely, a multi-scale network and a shape regression module. We perform an ablation study over CTW1500 to identify the contribution of these two components. Four models are trained as shown in Table 3. The first is 'Baseline' which refers to the original EAST model [Zhou et al., 2017] that regresses text pixels to four quadrilateral vertices. The second is 'Baseline+Multi-Scale' which uses EAST but includes the proposed multi-scale network structure. The third is 'Baseline+Shape Regression' that uses EAST but regresses to the nearest text boundary. The last is 'Multi-Scale Shape Regression' that fully implements both Multi-Scale and Shape Regression.

As Table 3 shows, the inclusion of multi-scale network alone improves the recall significantly with certain sacrifice of precision, and the inclusion of the shape regression alone improves both recall and precision clearly, leading to a $17 \mathrm{im}$ provement in $\mathrm{f}$-score. In addition, the inclusion of both multiscale network and shape regression module improves the fscore by over 21 beyond the baseline. Fig. 5 illustrate the ablation study where many missing and broken detection are correctly detected by the Multi-Scale+Shape Regression.

Acknowledgement: This work is funded by Ministry of Education, Singapore, under the project "A semi-supervised learning approach for accurate and robust detection of texts in scenes" (RG128/17 (S)).

\begin{tabular}{|l|c|c|c|}
\hline Methods & P & R & F-score \\
\hline \hline Baseline & 78.7 & 49.1 & 60.4 \\
Baseline+Multi-Scale & 72.8 & 60.8 & 66.3 \\
Baseline+Shape Regression & 82.8 & 72.1 & 77.1 \\
Multi-Scale Shape Regression & $\mathbf{8 5 . 0}$ & $\mathbf{7 8 . 3}$ & $\mathbf{8 1 . 5}$ \\
\hline
\end{tabular}

Table 3: Ablation study of the proposed technique over the dataset CTW1500 (P: precision; R: recall)

\subsection{Discussion}

The proposed method still faces certain constraints under several specific scenarios. First, it could fail while dealing with text lines that spatially overlap with each other as shown in the first row of Fig. 4 (e), largely due to the ambiguity in differentiating the central text region while text lines overlap with each other. Second, it could produce broken detection when characters in a word or text line are widely separated as shown in the last example image in the second row of Fig. 4 (e). Without text semantics, it's a common challenge to decide whether characters/letters belong to the same text line when they are widely separated.

\section{Conclusion}

This paper presents a novel multi-scale shape regression network that is capable of locating scene texts of different orientations, shapes and lengths accurately. The proposed method predicts dense text boundary points instead of sparse quadrilateral vertices that are prone to produce large regression errors while dealing with long text lines. In addition, this also enables accurate localization of scene texts of arbitrary orientations and curvatures whereas state-of-the-art techniques using quadrilaterals often include undesired background to the ensuing scene text recognition task. The multi-scale network extracts and fuses features at different scales which demonstrates superb tolerance to the text scale variation. Extensive experiments over several public datasets show the superior performance of the proposed technique. 


\section{References}

[Akkiraju et al., 1995] Nataraj Akkiraju, Herbert Edelsbrunner, Michael Facello, Ping Fu, EP Mucke, and Carlos Varela. Alpha shapes: definition and software. In Proceedings of the 1st International Computational Geometry Software Workshop, volume 63, page 66, 1995.

[Ch'ng and Chan, 2017] Chee Kheng Ch'ng and Chee Seng Chan. Total-text: A comprehensive dataset for scene text detection and recognition. In Document Analysis and Recognition (ICDAR), 2017 14th IAPR International Conference on, volume 1, pages 935-942, 2017.

[Deng et al., 2018] Dan Deng, Haifeng Liu, Xuelong Li, and Deng Cai. Pixellink: Detecting scene text via instance segmentation. In Thirty-Second AAAI Conference on Artificial Intelligence, 2018.

[Girshick, 2015] Ross Girshick. Fast r-cnn. In IEEE international conference on computer vision, 2015.

[Gupta et al., 2016] A. Gupta, A. Vedaldi, and A. Zisserman. Synthetic data for text localisation in natural images. In IEEE Conference on Computer Vision and Pattern Recognition, 2016.

[Hao et al., 2017] Zekun Hao, Yu Liu, Hongwei Qin, Junjie Yan, Xiu Li, and Xiaolin Hu. Scale-aware face detection. In The IEEE Conference on Computer Vision and Pattern Recognition (CVPR), volume 3, 2017.

[He et al., 2016] Kaiming He, Xiangyu Zhang, Shaoqing Ren, and Jian Sun. Deep residual learning for image recognition. In Proceedings of the IEEE conference on computer vision and pattern recognition, pages 770-778, 2016.

[He et al., 2017] Wenhao He, Xu-Yao Zhang, Fei Yin, and Cheng-Lin Liu. Deep direct regression for multi-oriented scene text detection. In IEEE International Conference on Computer Vision, pages 745-753, 2017.

[Huang et al., 2015] Lichao Huang, Yi Yang, Yafeng Deng, and Yinan Yu. Densebox: Unifying landmark localization with end to end object detection. arXiv preprint arXiv:1509.04874, 2015.

[Karatzas et al., 2015] Dimosthenis Karatzas, Lluis GomezBigorda, Anguelos Nicolaou, Suman Ghosh, Andrew Bagdanov, Masakazu Iwamura, Jiri Matas, Lukas Neumann, Vijay Ramaseshan Chandrasekhar, Shijian Lu, et al. Icdar 2015 competition on robust reading. In 2015 13th International Conference on Document Analysis and Recognition (ICDAR), pages 1156-1160, 2015.

[Kingma and Ba, 2014] Diederik P Kingma and Jimmy Ba. Adam: A method for stochastic optimization. arXiv preprint arXiv:1412.6980, 2014.

[Liao et al., 2018] Minghui Liao, Zhen Zhu, Baoguang Shi, Gui-song Xia, and Xiang Bai. Rotation-sensitive regression for oriented scene text detection. In Proceedings of the IEEE Conference on Computer Vision and Pattern Recognition, pages 5909-5918, 2018.

[Lin et al., 2017] Tsung-Yi Lin, Piotr Dollár, Ross B Girshick, Kaiming He, Bharath Hariharan, and Serge J Be- longie. Feature pyramid networks for object detection. In CVPR, volume 1, page 3, 2017.

[Long et al., 2018] Shangbang Long, Jiaqiang Ruan, Wenjie Zhang, Xin He, Wenhao Wu, and Cong Yao. Textsnake: A flexible representation for detecting text of arbitrary shapes. In The European Conference on Computer Vision (ECCV), 2018.

[Lyu et al., 2018a] Pengyuan Lyu, Minghui Liao, Cong Yao, Wenhao Wu, and Xiang Bai. Mask textspotter: An endto-end trainable neural network for spotting text with arbitrary shapes. In The European Conference on Computer Vision (ECCV), 2018.

[Lyu et al., 2018b] Pengyuan Lyu, Cong Yao, Wenhao Wu, Shuicheng Yan, and Xiang Bai. Multi-oriented scene text detection via corner localization and region segmentation. In Proceedings of the IEEE Conference on Computer Vision and Pattern Recognition, pages 7553-7563, 2018.

[Ronneberger et al., 2015] Olaf Ronneberger, Philipp Fischer, and Thomas Brox. U-net: Convolutional networks for biomedical image segmentation. In International Conference on Medical image computing and computerassisted intervention, pages 234-241, 2015.

[Shi et al., 2017] Baoguang Shi, Xiang Bai, and Serge Belongie. Detecting oriented text in natural images by linking segments. In 2017 IEEE Conference on Computer Vision and Pattern Recognition, pages 3482-3490, 2017.

[Singh and Davis, 2018] Bharat Singh and Larry S Davis. An analysis of scale invariance in object detection-snip. In Proceedings of the IEEE Conference on Computer Vision and Pattern Recognition, pages 3578-3587, 2018.

[Tian et al., 2015] Shangxuan Tian, Yifeng Pan, Chang Huang, Shijian Lu, Kai Yu, and Chew Lim Tan. Text flow: A unified text detection system in natural scene images. In Proceedings of the IEEE international conference on computer vision, pages 4651-4659, 2015.

[Tian et al., 2016] Zhi Tian, Weilin Huang, Tong He, Pan $\mathrm{He}$, and Yu Qiao. Detecting text in natural image with connectionist text proposal network. In European conference on computer vision, pages 56-72. Springer, 2016.

[Xue et al., 2018] Chuhui Xue, Shijian Lu, and Fangneng Zhan. Accurate scene text detection through border semantics awareness and bootstrapping. In European Conference on Computer Vision, pages 370-387, 2018.

[Yao et al., 2012] Cong Yao, Xiang Bai, Wenyu Liu, Yi Ma, and Zhuowen Tu. Detecting texts of arbitrary orientations in natural images. In 2012 IEEE Conference on Computer Vision and Pattern Recognition, pages 1083-1090, 2012.

[Yuliang et al., 2017] Liu Yuliang, Jin Lianwen, Zhang Shuaitao, and Zhang Sheng. Detecting curve text in the wild: New dataset and new solution. arXiv preprint arXiv:1712.02170, 2017.

[Zhou et al., 2017] Xinyu Zhou, Cong Yao, He Wen, Yuzhi Wang, Shuchang Zhou, Weiran He, and Jiajun Liang. East: an efficient and accurate scene text detector. In Proc. CVPR, pages 2642-2651, 2017. 